\title{
Impact agro écologique de simulation de culture transgénique de maïs résistant au glyphosate et effet répétitif d'un traitement herbicide sur la flore adventice en Cote d'ivoire
}

\author{
Maxime N. K. Boraud ${ }^{1 *}$, Emma AKÉ-AsSI ${ }^{1}$, N'Dja J. KaSSI ${ }^{1}$, Severin AKÉ ${ }^{1}$ \& Jacques GasqueZ ${ }^{2}$ \\ 'Université de Cocody Abidjan, UFR Biosciences, Laboratoire de Botanique, 22 bp 582 Abidjan 22, tel : 05703630 ; \\ 2Unité Mixte de Recherche de Biologie et Gestion des Adventices INRA 17 rue Sully BP 8651021065 Dijon cedex FRANCE \\ *Auteur pour les corrspondances (E-mail : boraudn@hotmail.com; maxime.boraud@univ-cocody.ci) \\ Reçu le 04-06-2009, accepté 06-05-2010.
}

\section{Résumé}

Les impacts agro écologiques des cultures transgéniques sont les flux de gènes entre les cultures de même nature et entre la culture transgénique et les adventices du milieu de culture. Entre autres menaces sur les écosystèmes du sol, on note la perturbation de la diversité floristique par l'utilisation répétée du glyphosate quand le transgène confère la tolérance à un herbicide. Une étude de simulation de culture transgénique résistante au glyphosate avec le maïs a permis de suivre les flux de pollen entre les cultures de maïs et entre la culture de maïs et les adventices. Cette étude a permis de suivre également l'effet du glyphosate sur les adventices pendant trois ans. En Côte d'Ivoire, ce flux de gènes peut être transporté sur de longues distances malgré l'existence de barrière naturelle. Cependant le flux de gènes entre le maïs transgénique et les adventices est beaucoup plus complexe et doit nécessairement remplir plusieurs conditions dont la proximité de l'adventice à la culture, le mode de reproduction et la simultanéité de la floraison entre l'adventice et la culture et la compatibilité génétique. L'utilisation répétée du glyphosate au même endroit entraîne une réduction importante de la diversité floristique et l'apparition de nouvelles adventices.

Mot clés : impacts agro écologiques, culture transgénique, flux de gènes, adventice, Côte d'Ivoire

\begin{abstract}
Agro-ecological impact of transgenic crop simulation of maize resistant to glyphosate and repetitive effect of a herbicide on the weed flora in Côte d'lvoire

The agro-ecological impacts of transgenic crops is gene flow between crops and similar between the transgenic crop and weeds of the culture land. Among other threats to soil ecosystems, there is disruption of plant diversity by the repeated use of glyphosate when the transgene confers tolerance to an herbicide. A simulation study of transgenic crop with glyphosate-resistant corn was used to follow the flow of pollen between corns and between corn and weeds. This study also follows the effect of glyphosate on weeds for three years. In Côte d'Ivoire, this gene flow may be transported over long distances despite the existence of natural barrier. But gene flow from transgenic maize and weeds is much more complex and has to fulfil several conditions including the proximity of the weed culture, mode of reproduction and the simultaneity of flowering between the adventitia and culture and genetic compatibility. Repeated use of glyphosate in the same place leads to a significant reduction of plant diversity and the emergence of new weeds.
\end{abstract}

Keywords: agro-ecological impacts, transgenic crop gene flow, weed, Côte d'lvoire 


\section{Introduction}

Les plantes transgéniques avec leurs énormes potentialités (rendements élevés, résistances aux maladies, aux herbicides, aux insectes et au stress hydrique) selon Jaglo-Ottosen et al., 1998, semblent représenter un atout en agriculture pour l'Afrique. Elles pourraient contribuer à la réduction de la pauvreté et à l'augmentation de la production agricole dans cette zone du monde souvent confrontée à la famine (Graham et Peter, 2008). La plupart des plantes transgéniques cultivées dans le monde confère la tolérance à un herbicide pour $68 \%$ des cultures (Clive, 2006).

Les organismes génétiquement modifiés traînent un cortège de polémiques et de risques décriés qui concernent l'environnement à travers le flux du transgène par le pollen vers les cultures non transgéniques et vers les adventices, et aussi le flux par les graines (Oury, 2006).

Les cultures transgéniques représenteraientelles un danger pour l'environnement?

Dans les parcelles abritant les cultures résistantes au glyphosate, il y a une réduction de la diversité floristique et une acquisition de la résistance par certaines mauvaises herbes du fait de l'utilisation répétée d'un même herbicide (Baerson et al., 2002 ; Baylis, 2000).

L'objectif de cette étude est d'identifier par simulation, les risques réels que présente une culture transgénique de maïs résistante à un herbicide sur les autres espèces végétales environnant en Côte d'Ivoire. Pour atteindre cet objectif, il a été réalisé :

- la dispersion de gènes d'une culture de maïs dans des conditions naturelles;

- le recensement de toutes les adventices présentes dans les différents agrosystèmes immédiats et l'effet d'un traitement herbicide à base du glyphosate sur ces adventices.

\section{Sites d'étude}

L'étude a été menée à Gagnoa et à Azaguié, deux localités ivoiriennes. Azaguié est situé à $30 \mathrm{~km}$ au Nord d'Abidjan, en zone forestière, dans la Région de l'Agneby. Les sols ferrallitiques issus des différentes roches sont tous fortement désaturés. Leur horizon humifère est peu épais, médiocrement pourvu en matière organique et plus acide que les horizons sous-jacents (Guillaumet et al., 1971). Azaguié connaît quatre saisons (Berron, 1978) : une grande saison de pluies (avril-mi-juillet), une petite saison sèche (mi-juillet-mi-septembre), une petite saison des pluies (mi-septembre-novembre) et une grande saison sèche (décembre-mars). La pluviométrie annuelle se situe entre 1600 et $2100 \mathrm{~mm}$ de pluie.

Gagnoa se situe au Centre-Ouest de la Côte d'Ivoire, dans la Région du fromager. Les sols ferrallitiques sont fortement désaturés caractérisés par la présence d'un horizon humifère peu épais et un horizon gravillonnaire peu développé (Avenard et al., 1971). On y rencontre aussi des sols hydromorphes représentés par des sols de bas fonds.

La pluviométrie annuelle est comprise entre 1400 et $2000 \mathrm{~mm}$ de pluie et par l'alternance de deux saisons des pluies et deux saisons sèches dont la plus longue a un déficit hydrique cumulé compris entre 250 et $500 \mathrm{~mm}$ et réparti sur 3 à 5 mois (Avenard et al., 1971).

\section{Matériel et méthodes}

\subsection{Matériel d'étude}

Le matériel d'étude est constitué de la flore adventice et des variétés de maïs de différentes couleurs de caryopses (violet et blanc). II s'agit des variétés: Ferké 7529 avec la nature génétique suivante: population sélectionnée, cycle de 120 jours, couleur des graines violette considérée comme la variété transgénique et TZBR Ecdana 3C2 avec la nature génétique : population sélectionnée, cycle de 110-120 jours, couleur des graines blanche considérée comme la variété conventionnelle biologique (Anonyme, 2008). Toutes ces variétés sont homozygotes en ce qui concerne la coloration de la graine. Le produit chimique utilisé est l'herbicide Kalach de « Calivoire » dont la matière active est le glyphosate.

\subsection{Méthodes d'étude}

\subsubsection{Etude des flux de gènes}

\subsubsection{Dispositif 1 : Suivi des flux de gènes entre champs voisins}

Sur une parcelle centrale de superficie $5 \mathrm{~m} \times 7 \mathrm{~m}$ 
est cultivée une variété de maïs à caryopses violets à raison de 15 pieds de maïs par ligne de culture avec au total 15 lignes, soit environ 225 pieds de maïs à caryopses violets considérés comme la source pollinisatrice. Sur chaque ligne de culture de la parcelle centrale, les semis sont distants de $30 \mathrm{~cm}$. Les lignes de cultures sont équidistantes de $50 \mathrm{~cm}$. Autour de cette parcelle sont mises en place quatre autres parcelles d'égales superficies $(10 \mathrm{~m} \times 7 \mathrm{~m})$ disposées aux quatre points cardinaux de façon contiguë à la parcelle centrale. A l'intérieur de chacune des parcelles, des lignes de cultures ont été semées d'une variété de maïs de caryopses blancs. Sur chaque ligne de culture également, les semis sont distants de $30 \mathrm{~cm}$. Les lignes de cultures sont équidistantes de $50 \mathrm{~cm}$. Les parcelles contiguës, au nombre de 4 , sont constituées chacune de 30 pieds de maïs par ligne de culture et en ont 21 lignes, soit 630 pieds de maïs par parcelle. Ce qui fait un total de 2520 pieds de maïs pour l'ensemble des quatre champs contigus. L'essai de maïs a été réalisé entre Avril et Août. La différence de taille entre la parcelle centrale (plus petite) et les parcelles contigües (plus grandes) est de montrer l'existence du flux de gènes entre cultures voisines semblables quelque soit la surface du champ émetteur. A la récolte, il a été compté par épi de maïs dans les 4 parcelles autour de la parcelle centrale et par ligne de culture, le nombre de caryopses violets obtenus dans les semis à caryopses blancs. Ces caryopses violets sont semés par la suite pour confirmer l'acquisition du caractère couleur violette.

\subsubsection{Dispositif 2 : Suivide flux de gènes entre champs de maïs séparés par un couvert végétal}

Deux parcelles de surface $7 \mathrm{~m} \times 5 \mathrm{~m}$ chacune ont été retenues pour les travaux. La première parcelle a été semée avec du maïs à caryopses blancs et l'autre avec du maïs à caryopses violets. Les deux parcelles sont séparées de $57 \mathrm{~m}$ par une bande de plantation d'arbres d'hévéa. Cette plantation qui sépare les deux parcelles est âgée de 15 ans, haute d'environ $25 \mathrm{~m}$, large de $50 \mathrm{~m}$ et étendue sur $150 \mathrm{~m}$. A la récolte, sur chaque ligne de culture de la parcelle semée de caryopses blancs, le nombre de caryopses violets présents dans les épis a été évalué. Les champs ont été semés en Avril et récoltés en Août 2005.

\subsubsection{Relevés floristiques}

Un inventaire exhaustif des adventices a été réalisé dans les champs de maïs réalisés et les cultures vivrières de Gagnoa et d'Azaguié autour des parcelles expérimentales selon l'échantillonnage stratifié (Godron, 1971 ; Daget et Godron, 1982). Chaque agro système constitue une unité d'échantillonnage. L'étude floristique réalisée dans les agrosystèmes paysans dans les Départements d'Azaguié et de Gagnoa a porté sur 884 relevés dont la moitié dans chaque localité.

\subsubsection{Traitement herbicide}

Les observations ont été réalisées en parcelles expérimentales constituées de jachères contiguës aux parcelles de culture de maïs. Un bloc de Fischer de (5) répétitions et (9) traitements (Guinochet, 1973), soit 45 placettes de $9 \mathrm{~m}^{2}$ chacune a été utilisé. Ces placettes n'ont pas été semées et ont toutes reçu des traitements herbicides (glyphosate) 3 fois par an (Mars-JuinSeptembre) durant 3 années consécutives (20052007). A l'intérieur de chaque placette, un relevé exhaustif des adventices selon l'échantillonnage stratifié a été réalisé avant et après le traitement herbicide. Les concentrations de glyphosate utilisées pour les traitements sont dans le Tableau 1. Le traitement [T9] étant témoin. Chaque concentration de glyphosate a été complétée avec l'eau de robinet pour obtenir un volume total de $5 \mathrm{I}$ qui a servi pour le traitement des 5 répétitions à l'aide d'un pulvérisateur à pression préalable constante ; ce qui correspond à 11 de volume de produit reçu pour chaque placette de $9 \mathrm{~m}^{2}$. 
Tableau 1 : Traitements et concentrations de glyphosate utilisées

\begin{tabular}{cccc}
\hline Traitement & $\begin{array}{c}\text { Concentration matière } \\
\text { active }(\mathrm{g} / \mathrm{ha})\end{array}$ & $\begin{array}{c}\text { Quantité de produit } \\
\text { utilisé }(\mathrm{ml})\end{array}$ & $\begin{array}{c}\text { Nombre de } \\
\text { placettes traitées }\end{array}$ \\
\hline T1 & 360 & 50 & 5 \\
T2 & 340 & 47 & 5 \\
T3 & 320 & 44,4 & 5 \\
T4 & 300 & 41,7 & 5 \\
T5 & 280 & 38,9 & 5 \\
T6 & 380 & 52,8 & 5 \\
T7 & 400 & 55,6 & 5 \\
T8 & 420 & 58 & 5 \\
\hline
\end{tabular}

\subsubsection{Analyse statistique}

Des statistiques élémentaires (moyenne, écart type, variance) ont été réalisées avec Excel de Microsoft pour suivre la répétition des caryopses de pollens à travers les lignes de cultures.

\section{Résultats}

\subsection{Suivi des flux de gènes}

\subsubsection{Champs voisins}

Les six premières lignes sont utilisées pour exploiter l'ensemble des résultats car au-delà, la dispersion de caryopses violets est peu visible et la moyenne d'apparition de ces caryopses sur les épis de caryopses blancs de maïs est comprise entre 0,14 et 0,43 (Tableau 2). Les moyennes sur les différentes lignes en fonction de l'orientation des champs indiquent que les champs qui ont reçu de forts taux de pollen en provenance de la source pollinisatrice constituée de la parcelle centrale (maïs à caryopses violets) sont ceux situés en direction Est et Ouest.

\subsubsection{Champs voisins séparés par une végétation}

La récolte a donné des caryopses violets dans les épis de maïs blanc de la ligne 1 à la ligne 5 (Tableau 3 ). Au delà de la ligne 5 , la moyenne de grain violet présent est comprise entre 0,25 et 0,37 . La ligne 5, qui est la plus éloignée (169 m de la source pollinisatrice) a reçu la plus importante quantité de pollen émise de la source (Tableau 4).

\subsection{Diversité floristique}

L'analyse floristique a permis d'identifier 260 espèces réparties entre 150 genres et 57 familles à Azaguié et 242 espèces réparties entre 145 genres et 48 familles à Gagnoa. Elle a donné un total de 302 espèces avec 200 espèces communes aux 2 sites de l'étude. A Azaguié, on dénombre 22 genres et 43 espèces appartenant aux Poaceae alors qu'à Gagnoa on obtient 23 genres et 39 espèces appartenant aux Poaceae.

\subsection{Dynamique des adventices et contrôle chimique}

Au cours des trois années d'investigation, 405 relevés ont été réalisés. L'application successive d'herbicide à base de glyphosate trois fois par an sur la même parcelle durant trois campagnes a agit sur la diversité floristique et a engendré une baisse de $2 / 3$ du nombre d'espèces et de $1 / 2$ des nombres de familles et de genres (Tableaux 5 et 6). L'efficacité de l'herbicide utilisé est de $80 \%$ pour les Traitements T1 à T5 et oscille entre 80 et $90 \%$ pour les traitements T6 à T8. L'utilisation répétée de l'herbicide favorise la sélection d'un certain nombre d'adventices telles que Commelina benghalensis, Euphorbia heterophylla, Imperata cylindrica et Ipomoea involucrata. 
Tableau 2 : Nombre de caryopses violets obtenus sur les huit premières lignes de culture dans les champs contigus

\begin{tabular}{|c|c|c|c|c|c|c|c|c|c|c|c|}
\hline \multirow[t]{2}{*}{ Direction } & \multirow{2}{*}{$\begin{array}{c}\begin{array}{c}\text { Lignes de } \\
\text { cultures }\end{array} \\
\text { L1 }\end{array}$} & \multicolumn{7}{|c|}{$\begin{array}{l}\text { Nombre de caryopses violets dans les épis par } \\
\text { pied de maïs récoltés }\end{array}$} & \multirow{2}{*}{$\begin{array}{c}\text { Moyennes } \\
1,71\end{array}$} & \multirow{2}{*}{$\begin{array}{c}\begin{array}{c}\text { Ecarts } \\
\text { types }\end{array} \\
2,37\end{array}$} & \multirow[t]{2}{*}{$\begin{array}{c}\text { Taux de } \\
\text { contamination }\end{array}$} \\
\hline & & 3 & 0 & 1 & 7 & 1 & 0 & 0 & & & \\
\hline \multirow{7}{*}{ Sud } & L2 & 81 & 71 & 17 & 4 & 0 & 21 & 41 & 33,57 & 29,62 & \multirow{7}{*}{4,94} \\
\hline & L3 & 0 & 0 & 16 & 1 & 3 & 51 & 28 & 14,14 & 17,93 & \\
\hline & L4 & 0 & 0 & 1 & 1 & 0 & 0 & 1 & 0,43 & 0,53 & \\
\hline & L5 & 0 & 0 & 1 & 0 & 0 & 0 & 1 & 0,29 & 0,49 & \\
\hline & L6 & 1 & 1 & 1 & 0 & 0 & 0 & 0 & 0,43 & 0,53 & \\
\hline & $\mathrm{L} 7$ & 0 & 0 & 0 & 0 & 0 & 0 & 1 & 0,14 & 0,38 & \\
\hline & L8 & 1 & 1 & 0 & 0 & 0 & 0 & 0 & 0,29 & 0,49 & \\
\hline \multirow{8}{*}{ Est } & L1 & 24 & 103 & 55 & 3 & 0 & 14 & 22 & 31,57 & 33,64 & \multirow{8}{*}{10,12} \\
\hline & L2 & 61 & 43 & 33 & 107 & 8 & 1 & 33 & 40,86 & 32,90 & \\
\hline & L3 & 11 & 83 & 81 & 24 & 3 & 0 & 0 & 28,86 & 34,49 & \\
\hline & L4 & 8 & 4 & 1 & 0 & 0 & 1 & 0 & 0,29 & 0,53 & \\
\hline & L5 & 8 & 7 & 5 & 6 & 2 & 0 & 0 & 4,00 & 3,32 & \\
\hline & L6 & 0 & 0 & 1 & 1 & 0 & 0 & 1 & 0,43 & 0,53 & \\
\hline & $\mathrm{L} 7$ & 1 & 0 & 1 & 0 & 1 & 0 & 0 & 0,43 & 0,53 & \\
\hline & L8 & 1 & 0 & 0 & 0 & 0 & 0 & 0 & 0,14 & 0,38 & \\
\hline \multirow{8}{*}{ Ouest } & L1 & 151 & 12 & 4 & 150 & 67 & 98 & 27 & 72,71 & 57,66 & \multirow{8}{*}{13,87} \\
\hline & L2 & 122 & 87 & 28 & 11 & 9 & 0 & 3 & 37,14 & 44,39 & \\
\hline & L3 & 109 & 98 & 4 & 17 & 1 & 1 & 6 & 33,71 & 44,52 & \\
\hline & L4 & 29 & 12 & 10 & 11 & 1 & 2 & 3 & 9,71 & 9,66 & \\
\hline & L5 & 25 & 20 & 12 & 10 & 1 & 1 & 2 & 10,14 & 9,62 & \\
\hline & L6 & 5 & 4 & 5 & 4 & 0 & 0 & 0 & 2,57 & 2,44 & \\
\hline & L7 & 0 & 0 & 0 & 0 & 0 & 0 & 1 & 0,14 & 0,38 & \\
\hline & L8 & 0 & 0 & 0 & 0 & 0 & 0 & 0 & - & - & \\
\hline \multirow{8}{*}{ Nord } & L1 & 0 & 0 & 3 & 14 & 0 & 1 & 0 & 2,57 & 4,78 & \multirow{8}{*}{3,44} \\
\hline & L2 & 1 & 7 & 1 & 23 & 18 & 9 & 2 & 8,71 & 8,08 & \\
\hline & L3 & 4 & 21 & 55 & 74 & 0 & 7 & 1 & 23,14 & 27,41 & \\
\hline & L4 & 0 & 0 & 0 & 0 & 1 & 0 & 1 & 0,29 & 0,49 & \\
\hline & L5 & 0 & 0 & 0 & 0 & 0 & 0 & 0 & - & - & \\
\hline & L6 & 1 & 1 & 0 & 0 & 0 & 0 & 0 & 0,29 & 0,49 & \\
\hline & L7 & 0 & 0 & 1 & 0 & 0 & 0 & 0 & 0,14 & 0,38 & \\
\hline & L8 & 0 & 0 & 0 & 0 & 0 & 0 & 0 & - & - & \\
\hline
\end{tabular}

$L$ - Lignes de culture de maïs

Tableau 3 : Nombre de caryopses violets présents par pied de maïs dans les lignes de cultures de la parcelle semée de caryopses blancs en essai discontinu

\begin{tabular}{ccrcccrccc}
\hline Lignes & \multicolumn{1}{c}{ Nombre de caryopses violets } & Totaux \\
\hline L1 & 0 & 0 & 0 & 1 & 0 & 9 & 0 & 0 & 10 \\
L2 & 0 & 0 & 0 & 0 & 3 & 0 & 1 & 4 & 4 \\
L3 & 5 & 1 & 3 & 0 & 0 & 11 & 0 & 0 & 20 \\
L4 & 0 & 0 & 7 & 1 & 1 & 1 & 1 & 0 & 11 \\
L5 & 3 & 13 & 4 & 0 & 0 & 33 & 0 & 0 & 53 \\
\hline
\end{tabular}

L - Lignes de culture de maïs de la parcelle semée de caryopses blancs 
Tableau 4 : Nombre de caryopses violets recensés en fonction à la distance de la source pollinisatrice

\begin{tabular}{ccc}
\hline $\begin{array}{c}\text { Lignes de cultures semées de } \\
\text { caryopses blancs }\end{array}$ & $\begin{array}{c}\text { Distance de la source } \\
\text { émettrice }(\mathrm{m})\end{array}$ & $\begin{array}{c}\text { Moyennes de caryopses violets } \\
\text { par épi de maïs blanc }\end{array}$ \\
\hline L1 & 167 & 1,3 \\
L2 & 167,5 & 1,0 \\
L3 & 168 & 2,5 \\
L4 & 168,5 & 1,4 \\
L5 & 169 & 6,6 \\
\hline
\end{tabular}

L - Lignes de culture de maïs

Tableau 5 : Diversité floristique liée au traitement herbicide

\begin{tabular}{l|ccc}
\hline \multirow{2}{*}{ Paramètres } & \multicolumn{3}{c}{ Nombre des paramètres/ année } \\
\cline { 2 - 4 } Familles & 2005 & 2006 & 2007 \\
Genres & 25 & 20 & 13 \\
\hline Espèces & 49 & 35 & 17 \\
\hline
\end{tabular}

Tableau 6 : Familles et espèces recensées pendant trois années après le traitement herbicide à base du glyphosate

\begin{tabular}{clccc}
\hline & & \multicolumn{3}{c}{ Nombre des espèces } \\
\cline { 3 - 5 } $\mathrm{N}^{\circ}$ & Famille & 2005 & 2006 & 2007 \\
\hline 1 & Amaranthaceae & 2 & 1 & 1 \\
2 & Asteraceae & 3 & 6 & 2 \\
3 & Caesalpiniaceae & 1 & 1 & - \\
4 & Commelinaceae & 3 & 2 & 1 \\
5 & Convolvulaceae & 2 & 5 & 2 \\
6 & Cyperaceae & 6 & 7 & 1 \\
7 & Euphorbiaceae & 5 & 4 & 2 \\
8 & Fabaceae & 2 & 2 & 1 \\
9 & Malvaceae & - & 1 & - \\
10 & Mimosaceae & 1 & - & - \\
11 & Poaceae & 13 & 9 & 3 \\
12 & Rubiaceae & 7 & 5 & - \\
\hline
\end{tabular}

\section{Discussion}

L'ensemble des inventaires sur les deux localités explorées a permis d'identifier un total de 302 espèces avec 200 espèces communes aux deux départements. Du point de vue de la diversité floristique, 10 familles comportant les Poaceae sont les mieux représentées dans chaque zone d'étude. A Azaguié, les Poaceae renferment 22 genres et 43 espèces et à Gagnoa 23 genres et 39 espèces. Mais toutes ces espèces appartenant aux Poaceae ne sont pas génériquement proche du maïs et ne font pas partie des ancêtres du maïs. II ne peut donc pas avoir d'échange de pollen par voie sexuée entre les cultures de maïs et les mauvaises herbes.

Les adventices recensées conservent la prédominance des trois grandes familles (Poaceae, Asteracecae, Fabaceae) citées dans la flore des plantes nuisibles des milieux cultivés. Les résultats de cette étude sont en conformité avec les observations de nombreux auteurs qui ont travaillé en région tropicale (Merlier, 1972; Akobundu, 1977; Terry, 1981; Hoffman, 1986; Traoré, 1987; Le Bourgeois, 1993 ; Boraud, 1998, 2000). L'adaptation des familles, à espèces 
nombreuses, à des milieux très différents s'explique par Maillet (1981). Certaines espèces, notamment celles appartenant à la famille des Asteraceae sont anémochores et peuvent ensemencer rapidement les milieux cultivés.

Il y a une grande variation entre les moyennes et les écarts types, ce qui indique que la dispersion des flux de pollen n'est pas homogène au niveau de tous les champs. La différence de réception du flux de pollen au sein des champs contigus semble être liée au vent soufflant en direction Ouest-Est et à la taille des individus. Un autre facteur très important qui peut expliquer cette différence de dépôt de pollen au niveau des pieds de maïs est la floraison qui s'est effectuée très souvent en vague et qui n'est pas uniforme au niveau des champs. A partir de la troisième ligne, le vent semble ne plus avoir trop d'effet, la moyenne des caryopses violets présents varie entre $12 \%$ et $35 \%$.

II y a une nette diminution de caryopses violets dans la parcelle considérée comme non transgénique (champ semé de maïs à caryopses blancs) et séparé de la source pollinisatrice par une végétation par rapport aux autres champs contigus.

Les caryopses de maïs violets qu'on retrouve dans le champ de maïs blancs malgré la barrière que constitue le champ d'hévéa peut s'expliquer par le fait que l'obstacle constitué par la haie naturelle a pu être contourné par le courant d'air qui dans un premier temps élève en hauteur les caryopses de pollens provenant de la source pollinisatrice avant de se déposer de façon aléatoire sur les zones cibles constituées des autres espèces voisines et compatibles avec les plantes productrices de ces pollens.

Au cours d'une étude de dispersion de pollen réalisée par Pleasants et al. (1990), $90 \%$ du pollen a été perdu dans les 5 premiers mètres et $99 \%$ à 50 mètres. Mais pour cette étude réalisée en Côte d' Ivoire, à plus de $150 \mathrm{~m}$ des fécondations liées au grain de pollen ont été obtenues. En France à $100 \mathrm{~m}$, il a été obtenu 0,4 $\%$ dans une direction et $18 \%$ dans une autre (Klein et al. 2001). De nombreuses études ont donné moins de $1 \%$ du pollen à $200 \mathrm{~m}$. Les expériences sur le taux d'hybridation inter variétale ont souvent donné un taux inférieur ou égal à $1 \%$ de 20 à 40 m (Bateman, 1947). Les études tendent à confirmer une très faible probabilité de fécondation (inférieure à $1 \%$ ) après $200 \mathrm{~m}$. Avec des pièges à pollen placés à $1,5 \mathrm{~m}$ de hauteur, Scoot (1970) a obtenu à $230 \mathrm{~m}$ de la source, 0,85 $\%$ de flux existant au centre du champ émetteur. Reheul (1987) a observé du pollen de la source à plus de $1000 \mathrm{~m}$. Jensen et Bogh (1941) ont obtenu des taux de croisement de $0,42 \%$ à $400 \mathrm{~m}$ et 0,11 $\%$ à $600 \mathrm{~m}$. Des faibles taux de fécondations peuvent encore exister même à de grandes distances $(4 \mathrm{~km})$ par suite du transport du pollen par les insectes (Thompson et al., 1999).

Le glyphosate est plus efficace sur les plantules de façon générale, tandis que les espèces en fructification ou en floraison sont beaucoup plus résistantes, ce qui fait que 100\% d'efficacité a été rarement atteint. Par rapport aux espèces caractéristiques du milieu, le glyphosate, quelle que soit la dose utilisée permet une sélection en éliminant les adventices moins résistantes au profit des plus robustes. II y a donc la prolifération et le développement de certaines espèces qui au départ de l'expérimentation n'étaient pas présentes ou dominantes par rapport aux autres adventices. II s'agit de Imperata cylindrica, Ipomoea involucrata, Euphorbia heterophylla et Commelina benghalensis. Deux espèces résistent particulièrement à l'effet de l'herbicide (Ipomoea involucrata et Commelina benghalensis), elles ne s'assèchent pas complètement quel que soit le stade végétatif auquel l'application de l'herbicide est faite, contrairement aux espèces telles que Imperata cylindrica et Euphorbia heterophylla qui subissent l'effet de l'herbicide avant de reprendre leur croissance végétative. II s'agit peut être d'une résistance de ces adventices suite à l'utilisation répétée du glyphosate.

\section{Conclusion}

Cette étude de simulation permet d'affirmer avec certitude que les flux de gènes entre la culture de maïs transgénique résistant au glyphosate et la culture biologique conventionnelle voisines sont réels. La contamination entre ces deux types de cultures peut atteindre $13,87 \%$ en fonction de la proximité des champs, rendant transgénique la culture conventionnelle. Malgré la présence de barrière naturelle constituée par la végétation lors de la simulation, la contamination des cultures conventionnelles par la culture transgénique est 
possible mais à des taux moindres et acceptables. Cependant les flux de gènes entre le maïs transgénique et les mauvaises herbes sont peu probables par voie sexuée parce que le cortège de mauvaises herbes en Cote d'Ivoire ne contient pas d'adventices proches par le genre ou de mêmes ancêtres que le maïs.

L'utilisation répétée d'un herbicide à base de glyphosate réduit considérablement le nombre de mauvaises herbes, donc la biodiversité. Cependant les résultats révèlent également que l'utilisation répétée de l'herbicide favorise la sélection d'un certain nombre d'adventices telles Commelina benghalensis, Euphorbia heterophylla, Imperata cylindrica, Ipomoea involucrata qui se développent et prospèrent par rapport aux autres mauvaises herbes du milieu. II pourrait s'agir de développement de résistance au glyphosate de ces adventices par une utilisation répétée de cet herbicide.

\section{Références citées}

Akobundu I.O., 1977. Advances in weed control in conventional and no-tillage maize. Proceedings of the Seventh Conference of the Weed Science Society of Nigeria:10-20.

Anonyme, 2008. Catalogue Ouest africain des espèces et variétés végétales, CDEAO, UEMOA, CILSS, FAO, 107 pages.

Avenard, Guillaumet J. L. Adjanahoun E. \& Perraud. A. 1971. Le milieu naturel de la Côte d'lvoire. Mémoire ORSTOM, $N^{\circ}$ 50, Paris : 391 p.

Bateman A.J., 1947. Contamination in seed crops. I. Insect pollinisation. J. Genet.48: 257-275.

Baerson S.R., Damian J.R., Minhtien T., Yongmei F., Nancy A.B. \& Gerald M.D., 2002. Glyphosate-Resistant Goosegrass. Identification of a Mutation in the Target enzyme 5-Enolpyruvylshikimate-3phosphate synthase, Plant physiol, Vol 129, pp: $1265-1275$.

Baylis A.D., 2000. Why glyphosate is a global herbicide: strengths, weaknesses and prospects. Pest Management Science 56, 299-308.

Boraud N. K.M., 2000. Etude floristique et Phytoecologique des adventices des Complexes sucriers de Ferké 1 et 2, de Borotou- koro et de Zuénoula, en Côte d'lvoire. Thèse de Doctorat $3^{\text {ème }}$ cycle, Université de Cocody, $184 \mathrm{p}$.

Boraud N. K.M., Aman k. G., 1998. Les principales adventices de la canne à sucre en Côte d'Ivoire : similitudes et différences. XVII ${ }^{\text {ème }}$ conf. sur la lutte contre les mauvaises herbes Dijon (France). : 1173-1179, 7-9 décembre 1998.

Clive James, 2006. Global area of biotech crops. Million Hectares (1996 to 2006). http:// www.isaaa.org/Resources/Publication du 15 janvier 2007.

Hoffman G., 1986. Caractérisation de la flore adventice de deux villages du terroir de Katiola (Côte d'lvoire). Mémoire IAT ESATCNEARC-Montpellier, 51p.

Graham B. and Peter B., 2008. Global impact of Biotech crops : Socio-Economic and Environnemental effects, 1996-2006. Agbioforum, 11(1): 21-38.

Jaglo-Ottosen K., Gilmour S., Zarka D., Schabengerger., Thomas-Show M., 1998. Arabidiopsis CBF1 overexpression induces COR genes and enchanges free-zing tolerance. Science 280 : 104-106.

Jensen I., Bogh H., 1941. On conditions influencing the danger of crossing in case of wind pollinated cultivated plants. Tidsskrift for planteavl, 46: 238-266.

Klein E., Lavigne C., Foueillassar X., Laredo C., Gouyon P.H., 2001. Aip OGM et environnement, Edition Inra, Paris, $156 \mathrm{p}$.

Le Bourgeois Th., 1993. Les mauvaises herbes dans la rotation cotonnière au Nord Cameroun (Afrique). Amplitude d'habitat et degrés d'infestation. Phénologie. Thèse USTML, 204 p. + annexes.

Norris C.E., Sweet J.B., Cockley A.R., 2002. Gene flow by cross pollinisation between large adjacent blocks of spring oilseed rape (Brassica napus) at two farm scale sites. J. Aerosol Sci., Vol. 22, No 4, pp. 467-477.

Maillet J., 1981. Évolution de la flore adventice dans le Montpelliérain sous la pression des techniques culturales. Thèse de Docteur ingénieur. USTL-Montpellier, 200 p. 
Merlier H., 1972. Étude phénologique des espèces de jachère du centre Sénégal (synthèse). Agronomie Tropicale, Vol. XXVII, $\mathrm{n}^{\circ} 12: 1229-1252$.

Oury J.P., 2006. La querelle des OGM. Science, Histoire et Société, Edition PUF, 303 p.

Pleasants J. M., 1990. Evidence for Short-Distance Dispersal of Pollinia in Asclepias syriaca L. Functional Ecology, Vol. 5, No. 1 : 75-82.

Rehuel D., 1987. Ruimtelijke isolatie in de plantenveredeling. Landbouwtijdschrift, 40: 5-15.

Scott R.K., 1970. The effect of weather on the concentration of pollen within sugar beet crops. Annals of Application of Biologie, 66: 119-127.

Simpson E.C., Norris C.E., Law J.R., Thomas J.E. \& Sweet J.B. 1999. Gene flow in genetically modified herbicide tolerant oilseed rape (Brassica napus) in the UK édition BCPC, 172 p.
Terry P.J., 1981. Weeds and their control in Gambia. trop. Pest Manag. 27: 44-52.

Thompson C.E., Squire G., Mackay G.R., Bradshaw J.E. Crawford J. \& Ramsey G., 1999. Regional patterns of gene flow and its consequences for GM oilseed rape. In Gene flow and agriculture: relevance for transgenic crops. Lutman P. (Ed.) BCPC Symposium Proceedings. $300 \mathrm{p}$.

Timmons A.M., O'Brien E.T., Charters Y.M., Dubbels S.J. \& Wilkinson M.J., 1995. Assessing the risks of wind pollination from fields of genetically modified Brassica napus, var. oleifera. Euphytica. 85 : 417-423.

Traoré H., 1991. Influence des facteurs agroécologiques sur la constitution des communautés adventices des cultures céréalières (sorgho, mil, maïs) du BurkinaFaso. Thèse doctorat, USTL, Montpellier II, $180 \mathrm{p}$. 